\title{
Antigenic stimulation is more efficient than LPS in inducing nitric oxide production by human mononuclear cells on the in vitro granuloma reaction in schistosomiasis
}

D.M. Oliveirat, D.N. Silva-Teixeira ${ }^{1}$ R. Araújo-Filho ${ }^{3}$ and A.M. Goes ${ }^{1}$

\author{
${ }^{1}$ Departamento de Bioquímica e Imunologia, Instituto de Ciências Biológicas, \\ Universidade Federal de Minas Gerais, Belo Horizonte, MG, Brasil \\ 2Departamento de Medicina Veterinária, Faculdade de Veterinária, \\ Universidade Estadual do Ceará, Fortaleza, CE, Brasil \\ ${ }^{3}$ Departamento de Biologia, Centro de Ciências da Saúde, \\ Universidade de Fortaleza, Fortaleza, CE, Brasil
}

\section{Correspondence \\ A.M. Goes \\ Departamento de Bioquímica \\ e Imunologia, ICB, UFM G \\ 30161-970 Belo Horizonte, MG \\ Brasil \\ Fax: +55-31-441-5963 \\ E-mail: goes@ mono.icb.ufmg.br \\ Presented at the Meeting \\ "NO Brazil, Basic and Clinical \\ Aspects of Nitric $O$ xide", \\ Foz do Iguaçu, PR, Brazil, \\ March 10-13, 1999. \\ Research supported by CNPq, PRONEX, CAPES-PICDT, PRPq-UFMG and FAPEMIG}

Received June 17, 1999

Accepted September 8, 1999

\section{Abstract}

Nitric oxide (NO) is an extremely important and versatile messenger in biological systems. It has been identified as a cytotoxic factor in the immune system, presenting anti- or pro-inflammatory properties under different circumstances. In murine monocytes and macrophages, stimuli by cytokines or lipopolysaccharide (LPS) are necessary for inducing the immunologic isoform of the enzyme responsible for the high-output production of NO, nitric oxide synthase (iNOS). With respect to human cells, however, LPS seems not to stimulate NO production in the same way. Addressing this issue, we demonstrate here that peripheral blood mononuclear cells (PBMC) obtained from schistosomiasis-infected patients and cultivated with parasite antigens in the in vitro granuloma (IVG) reaction produced more nitrite in the absence of LPS. Thus, LPS-induced nitrite levels are easily detectable, although lower than those detected only with antigenic stimulation. Concomitant addition of LPS and L-N-arginine methyl ester (L-NAME) restored the ability to produce detectable levels of nitrite, which had been lost with L-NAME treatment. In addition, LPS caused a mild decrease of the IVG reaction and its association with LNAME was responsible for reversal of the L-NAME-exacerbating effect on the IVG reaction. These results show that LPS alone is not as good an NO inducer in human cells as it is in rodent cells or cell lines. Moreover, they provide evidence for interactions between LPS and $\mathrm{NO}$ inhibitors that require further investigation.
Key words

- Nitric oxide

- LPS

- Schistosomiasis infection

- In vitro granuloma 


\section{Introduction}

Nitric oxide (NO) is an extremely important and versatile messenger in biological systems. It has been identified as a cytotoxic factor in the immune system and as an antior pro-inflammatory element under different circumstances (1). Since inflammation is not one single process but a complex series of processes each characterized by different cellular populations, extracellular components and mediators, a body of evidence is also accumulating to indicate a role for $\mathrm{NO}$ as a key mediator of inflammation (1).

The statement that immune cells, including activated macrophages, monocytes and almost any type of cell can release NO in response to stimuli by cytokines or lipopolysaccharide (LPS) has long been accepted (2) and not questioned, especially concerning murine models and cell lines (3). However, LPS does not stimulate NO production in human systems, in the same way as it does in rodent cells (4). LPS, which is a constituent of bacterial outer membrane, has been recognized by many investigators as the prototype macrophage activator (5).

The inducible immunologic isoform of the enzyme responsible for the high-output production of $\mathrm{NO}$, nitric oxide synthase (iNOS), in human monocytes or macrophages is most readily observed in patients with infectious or inflammatory diseases. Sustained production of NO endows macrophages with cytostatic or cytotoxic activity against viruses, bacteria, fungi, protozoa, helminths and tumor cells. Although the highoutput NO pathway probably evolved to protect the host from infection, suppressive effects on lymphocyte proliferation and damage to other normal host cells confer upon iNOS the same protective/destructive duality inherent in every other major component of the immune response (6).

Infection with Schistosoma mansoni induces humoral $\mathrm{T}$ cell-mediated responses that result in granulomatous inflammatory disease around the parasite eggs (7). In this sense, it is extremely valid to use the in vitro granuloma (IVG) reaction which mimics an inflammatory response like that seen in schistosomiasis. We use peripheral blood mononuclear cells (PBMC), obtained from either S. mansoni-infected or non-infected individuals, and stimulate these cells with parasite antigenic preparations conjugated to polyacrylamide beads (8).

Recently, we have reported that NO inhibition by L-N-arginine methyl ester (L-NAME) acted by exacerbating the IVG reaction (9). This led us to determine whether this effect would persist under additional circumstances. Thus, we tried a second known stimulus, LPS, in order to create a supposedly better environment for NO release, at the same time that we tested the combined effects of L-NAME and LPS on the IVG reaction. Prior to focusing on the exact consequence of added stimulus versus antigenic stimulation during infection on effective in vitro NO release, we determined the ability of an exogenous stimulus (LPS) and of antigenic stimulation (S. mansoni preparations) to induce NO production by human mononuclear cells. The results presented here show that LPS is not a good NO inducer in human cells as it is in rodent cells or cell lines.

\section{Material and Methods}

\section{Reagents}

L-NAME and bacterial LPS (phenolextracted E. coli serotype 0128:B12) were purchased from Sigma Chemical Co. (St. Louis, MO, USA).

\section{Study population}

Ten schistosomiasis mansoni patients were selected based on parasitological stool examinations (10) in the Santa Luzia district (a known endemic area) through a cooperation program among Federal University of Minas Gerais (UFMG), Brazilian National 
Health Foundation (FNS) and Santa Luzia Municipal Health Service. The human subject Ethics Committee of Brazil has approved the patient protocols used throughout this study. Three healthy, uninfected individuals (normal controls) were selected among volunteers.

\section{Cell preparations}

PBMC were isolated from heparinized blood $(20 \mathrm{U} / \mathrm{ml}$ heparin) of patients with chronic intestinal schistosomiasis mansoni and uninfected controls by Ficoll diatriazoate density gradient centrifugation (LSM, Organon Teknica, Charleston, SC, USA) (11). PBMC were suspended in RPMI 1640 culture medium (Sigma) containing 1.6\% Lglutamine, $300 \mathrm{U} / \mathrm{ml}$ penicillin, $0.3 \mathrm{mg} / \mathrm{ml}$ streptomycin and $10 \%$ heat-inactivated human $\mathrm{AB}^{+}$serum, and then counted on a Neubauer chamber.

\section{S. mansoni antigens conjugated to polyacrylamide beads}

Polyacrylamide beads (PB), 40-60 $\mu \mathrm{m}$ in diameter (Bio-Gel P-4, Bio-Rad, Richmond, CA, USA), were washed in $0.5 \mathrm{M}$ bicarbonate buffer as previously described (8). The beads were mixed with $20 \mathrm{mg}$ of $S$. mansoni antigens obtained from eggs (SEA) and adult worms (SWAP) according to previously described techniques (12). Purified protein derivative (PPD) from Mycobacterium tuberculosis was purchased from Connaught Laboratories (Ontario, Canada) and was used as a positive control for the IVG reaction, since the Brazilian population is BCG-vaccinated and reacts to PPD, whereas PB alone (not conjugated to any antigen) was the negative control specific for the in vitro reaction.

\section{In vitro granuloma reaction}

The reaction was carried out utilizing antigen-coated beads as previously described
(12). Briefly, 200 polyacrylamide beads conjugated (or not) to $S$. mansoni antigens (PBSEA and PB-SWAP) or to M. tuberculosis antigen (PB-PPD) were added to the bottom of 96-well tissue culture plates together with $3 \times 10^{5}$ cells/well in distinct rows for each of the four treatments: a) presence of L-NAME $(1 \mathrm{mM}), \mathrm{b})$ presence of LPS $(1 \mu \mathrm{g} / \mathrm{ml}), \mathrm{c})$ presence of both LPS and L-NAME, and d) none of them, i.e., no addition. The PBMC were cultivated in a final volume of $200 \mu$ of RPMI with $10 \%$ heat-inactivated $\mathrm{AB}^{+}$human serum. Each experimental and control group was set up in triplicate and maintained at $37^{\circ} \mathrm{C}$ in a $5 \% \mathrm{CO}_{2}$ incubator. Cellular reactivity was determined by morphological observations of cellular migration and adherent cell layers surrounding the beads, using a phase-contrast inverted tissue culture microscope (Nikon TMS). Two hundred separate determinations of cellular reactivity were made for each experimental group. A numerical score equivalent to the following classification was assigned to each in vitro cell/bead reaction observed: 1) absence of cells binding to the beads; 2) less than 5 cells binding to the beads; 3 ) more than 5 cells binding to the beads; 4) more than 5 cells binding to the beads accompanied by circumoval mononuclear cell migration; 5) adherent cell monolayer attached to the beads accompanied by circumoval mononuclear cell migration; 6) multiple cell layers surrounding the beads accompanied by mononuclear cell migration (12).

The total score was then summed and the resultant mean expressed as the granuloma index (GI). Granulomatous reactivity to PBSEA, PB-SWAP and to a positive control (PB-PPD) was compared to the nonspecific binding of activated macrophages against polyacrylamide beads not conjugated to antigen (PB).

\section{Nitrite determination}

Nitrite concentration in culture, a meas- 

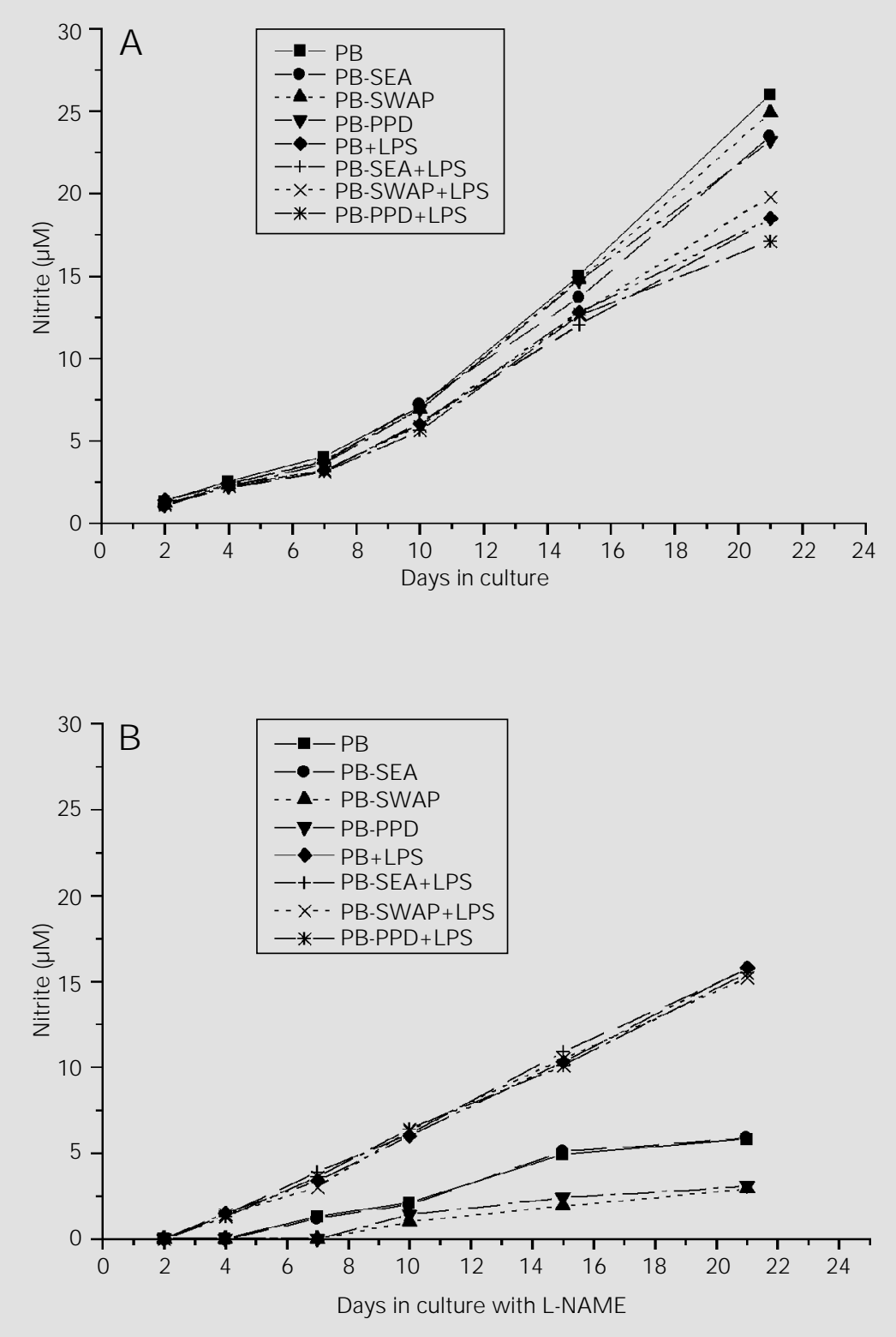

Figure 1 - Panel A, Effect of LPS on kinetics of NO production by PBMC from schistosomiasis patients during the in vitro granuloma (IVG) reaction. Cells $\left(3 \times 10^{5} /\right.$ well) were cultivated with polyacrylamide beads coated with S. mansoni (PB-SEA and PB-SWAP) or with $M$. tuberculosis (PB-PPD) antigens or not coated with any antigen (PB). The results are reported as nitrite concentrations $(\mu \mathrm{M})$ as a function of time and represent the mean of $\mathrm{N}=10$. The standard deviation did not exceed $15 \%$. All experiments were carried out in triplicate. $\mathrm{P}<0.05$ was taken as the limit of significance. Panel B, Inhibition of NO production by L-Narginine methyl ester (L-NAME) and its reversal by LPS and kinetics of NO production by PBMC from schistosomiasis patients during the IVG reaction. Cells $(3 \times 105 /$ well) were cultivated with PB-SEA, PB-SWAP, PB-PPD or PB alone and with L-NAME (1 mM) or with combined LPS $(1 \mu \mathrm{g} / \mathrm{ml})$ and L-NAME $(1 \mathrm{mM})$. The lower lines show the inhibited nitrite release in the presence of only L-NAME while the upper lines show restored nitrite release in presence of both LPS and L-NAME. urement of NO synthesis, was assayed by a standard Griess reaction adapted to microplates (13). The Griess reagent was prepared by mixing equal volumes of sulfanilamide $\left(1.5 \%\right.$ in $\left.5 \% \mathrm{H}_{3} \mathrm{PO}_{4}\right)$ and naphthylethylene diamine dihydrochloride $\left(0.1 \%\right.$ in $\left.\mathrm{H}_{2} \mathrm{O}\right)$. A volume of $50 \mu \mathrm{l}$ of this reagent was mixed with $50 \mu \mathrm{l}$ of tested supernatant and incubated at room temperature for $10 \mathrm{~min}$. Absorbance of the chromophore formed was measured at $540 \mathrm{~nm}$ using an automated microplate reader (Multiskan MCC/340, Labsystems, Helsinki, Finland). Nitrite concentrations were calculated by means of a $\mathrm{NaNO}_{2}$ standard curve and data were expressed as $\mu \mathrm{M}$ nitrite (14).

\section{Data analysis}

Data were analyzed statistically by the Student $t$-test, with the level of significance set at $\mathrm{P}<0.05$.

\section{Results}

\section{Effects of LPS on NO release and on its inhibition by L-NAME}

We have previously established that PBMC from $S$. mansoni-infected and noninfected individuals are able to produce NO when properly stimulated in the IVG microreaction (9). At that time and now, we also inhibited NO production by adding LNAME to the culture, a fact that exacerbated the IVG reaction, as reported earlier (9). In the present study, we used the same procedure to set up the experiments using LPS as a concomitant stimulus, and the data obtained are shown in Figure 1 (Panels A and B). Supernatants were periodically analyzed for nitrite determination on the $2 \mathrm{nd}, 4 \mathrm{th}, 7 \mathrm{th}$, 10 th, 15 th and 21 st days of culture. There was a general time-dependent increase in the amounts of nitrite detected. Figure 1A shows that nitrite detection in rows with LPS added or in rows without any addition (neither LPS 
nor L-NAME) was not significantly different, although LPS addition generally led to lower nitrite concentrations. On the other hand, when LPS was added together with LNAME, this combination restored the ability (lost by L-NAME treatment) of PBMC to release detectable levels of nitrite (Figure 1B). Comparison of NO production between groups with or without LPS showed a decrease in nitrite levels in the groups with LPS added, whereas in groups with L-NAME added, LPS addition did stimulate NO production (Figure 1A and B).

\section{LPS stimulus does not affect the IVG reaction, but, when associated with L-NAME, it reverses the IVG-exacerbating action of L-NAME}

Reactivity of cells (PBMC), from infected and uninfected individuals, cultivated with polyacrylamide beads conjugated to $S$. mansoni antigens (PB-SEA and PB-SWAP), to M. tuberculosis antigen (PB-PPD) or not conjugated to any antigen ( $\mathrm{PB}$ alone) is demonstrated in Figures $2 \mathrm{~A}$ and $\mathrm{B}$ and $3 \mathrm{~A}$ and $\mathrm{B}$. Cells to which only LPS was added (Figure 2B) showed slightly lower GI values than those with no LPS added (Figure 2A). Addition of L-NAME to IVG cultures exacerbated the IVG reaction as shown by the increase in GI values (Figure 3A). Association of LPS and L-NAME in culture, as depicted in Figure 3B, produced results similar to those seen in Figure 2A, where neither was added to the culture. This event demonstrates that LPS reverses the IVG-exacerbating property shown by L-NAME.

\section{Discussion}

The role of the L-arginine/NO pathway in human monocytes and macrophages has been highly debated $(4,15)$. Recent evidence suggests that human monocytes express the inducible form of NOS (iNOS) in response to LPS (16). The active component of endo-

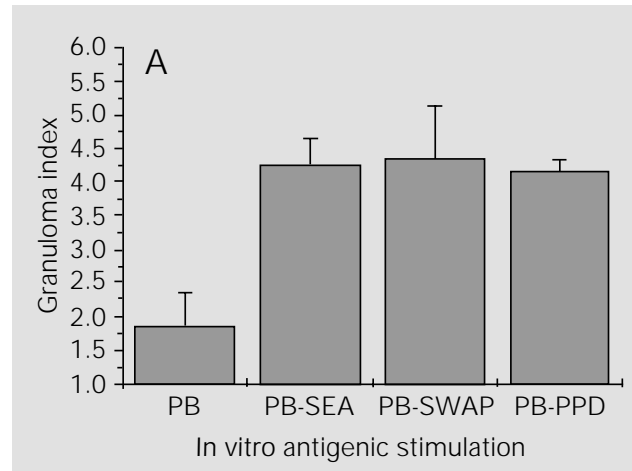

Figure 2 - Panel A, In vitro granuloma (IVG) reaction of PBMC from schistosomiasis patients. Cells $\left(3 \times 10^{5} /\right.$ well $)$ were cultivated with polyacrylamide beads coated with S. mansoni (PB-SEA and PB-SWAP) or M. tuberculosis (PB-PPD) antigens or not coated with antigen (PB). The results are expressed as granuloma index (GI) evaluated on the 7th day of IVG culture and represent the mean $\pm S D$ of $N=10$. All experiments were carried out in triplicate. $\mathrm{P}<0.05$ was taken as the limit of significance. Panel $B$, Effect of lipopolysaccharide (LPS) on the IVG reaction of PBMC from schistosomiasis patients. Cells $\left(3 \times 10^{5} /\right.$ well) were cultivated with PB-SEA, PBSWAP, PB-PPD or PB alone. LPS addition to IVG culture $(1 \mu \mathrm{g} / \mathrm{ml})$ did not significantly change the $\mathrm{Gl}$ values (as those presented in Figure $2 \mathrm{~A}$ ), but generally there was a mild decrease in the IVG reaction as shown by lower mean Gls compared to Figure 2A. 
Figure 3 - Panel A, Effect of L-Narginine methyl ester (L-NAME) in increasing the in vitro granuloma (IVG) reaction. The granuloma index $(\mathrm{Gl})$, ranging from 1 to 6 , is shown as a function of tested antigenic stimulation (PB, PB-SEA, PB-SWAP and PB-PPD) in the presence of L-NAME (1 $\mathrm{mM})$. The results are reported as $\mathrm{Gl}$ evaluated on the 7th day of IVG culture. There was an evident general increase in $\mathrm{Gl}$ values for all tested antigens to which L-NAME was added as compared to the results obtained with only LPS, with LPS plus L-NAME or without any addition, as shown in Figures $2 \mathrm{~A}$, 2B and 3B. Data are expressed as mean $\pm \mathrm{SD}$ of $\mathrm{N}=10$. All experiments were carried out in triplicate. $\mathrm{P}<0.05$ was taken as the limit of significance. Panel B, Effect of combined LPS and LNAME on the IVG reaction. The $\mathrm{Gl}$, ranging from 1 to 6 , is shown as a function of tested antigenic stimulation (PB, PB-SEA, PBSWAP and PB-PPD) in the concomitant presence of L-NAME $(1 \mathrm{mM})$ and LPS $(1 \mu \mathrm{g} / \mathrm{ml})$. The results are reported as $\mathrm{Gl}$ evaluated on the 7th day of IVG culture. As a whole, there was a significant decrease in $\mathrm{Gl}$ values for all tested antigens, as compared to data shown in Figure 3A (with only L-NAME addition), meaning that the exacerbating effect of L-NAME on the IVG reaction was reduced by LPS addition in culture.

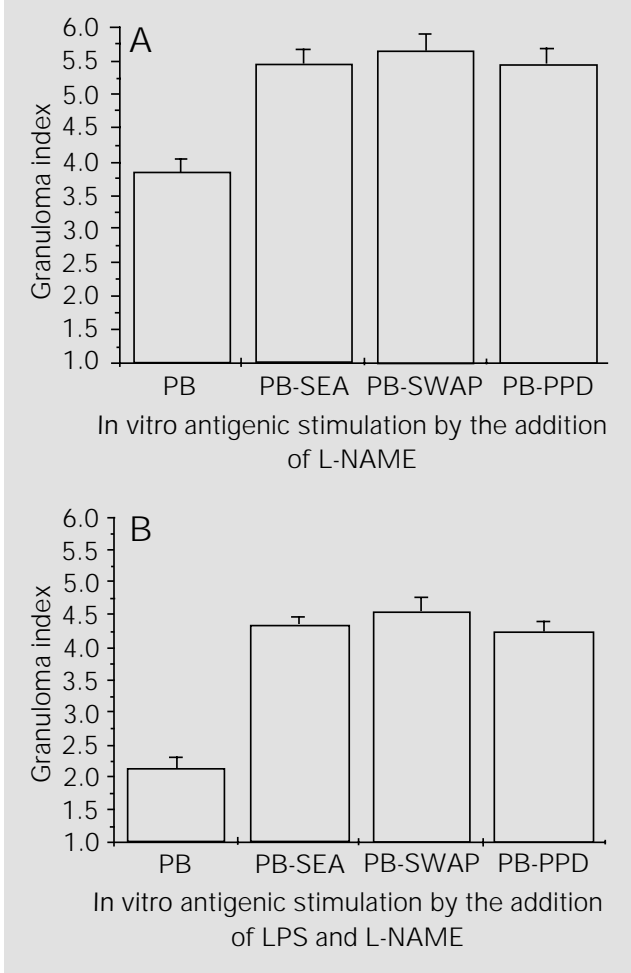

functions. Using this approach, many reports indicate that NO inhibition might have beneficial and/or deleterious effects on a variety of animal models of inflammatory or infectious diseases $(19,20)$. Although the cytotoxic activities of NO have been extensively described, several reports convincingly demonstrate a protective role for $\mathrm{NO}$ (21). However, in human pathophysiology, particularly in human monocytes and macrophages, the role of NOS remains elusive $(15,16)$. In our own experiments, we have seen that L-NAME addition to IVG culture is responsible for an exacerbation of the IVG reaction which appears to indicate at least an indirect anti-inflammatory role for NO.

With respect to LPS-induced iNOS activation, some investigators have reported that at least $500 \mathrm{ng}$ of LPS per $\mathrm{ml}$ is required to induce NO from RAW 264.7 cells, and this amount is reduced to only $500 \mathrm{pg} / \mathrm{ml}$ when IFN $\gamma$ is present (22), supporting the view that, even in this system, LPS alone is not so efficient. Yet, this macrophage cell line
(RAW 264.7) undergoes NO-dependent apoptosis after being stimulated with a combination of LPS plus IFN $\gamma$, while L-NMMA has been shown to exert cytoprotective and antiapoptotic effects on these cells $(19,23)$. Albeit in our study we did not specifically check the occurrence of apoptosis, there was seemingly no differences in cell viability among the four treatments tested (LPS, LNAME, LPS plus L-NAME, or none of them). Other studies have reported that endotoxin induces a progressive loss of vascular tone which correlates with the expression of a $\mathrm{Ca}^{2+}$-independent NOS in the vasculature. L-NMMA restores the loss of vessel tone and prevents the hyporesponsiveness to vasoconstrictor agents in endotoxin-treated isolated vessels in vitro (24) and, together with L-NAME, has been shown to restore blood pressure in animal models of endotoxin or septic shock (25). These indications of a direct interaction between LPS and L-arginine analogues agree with our results, which are consistent with the apparent antagonist effects of LPS and L-NAME in inducing NO production and in exacerbating the IVG reaction. The LPS stimulus clearly interfered with the expected effect of L-NAME on the IVG reaction both in terms of nitrite release and granuloma index values.

It is known that the most effective means of inducing macrophage activation is through combined stimulation with a priming and a triggering agent. Priming agents such as IFN $\gamma$ potentiate the macrophage response to a triggering agent like LPS (26). It is assumed that the order in which macrophages encounter immune and inflammatory stimuli can profoundly influence the extent to which $\mathrm{NO}$ synthesis is induced (26). Under the present conditions, we did not use IFN, but we used antigenic stimulation which turned out to be a potent priming and triggering agent, supplanting LPS as an inducer of NO production. Perhaps this was due to the attributes of the model we employ, the IVG reaction which, by itself, constitutes a continuous 
and progressive immune/inflammatory process. IVG begins with reaction of fresh PBMC and keeps them in differentiation processes in vitro until they become macrophages, multinucleated giant and epithelioid cells.

A few reports have investigated the activation mechanisms of the NOS or arginase pathway. One probable factor involved is the presence of bacterial antigens. In a recent investigation, LPS from nontoxic or detoxified sources specifically triggered the arginase pathway in murine bone marrow-derived macrophages and NOS was induced solely by toxic LPS (27). Another point to be taken into account is the influence of serum factors in culture medium. A recent study reported that LPS had a more dramatic stimulatory effect on NO induction if the levels of serum $\mathrm{NO}_{2}{ }^{-} / \mathrm{NO}_{3}{ }^{-}$preexisting in Corynebacterium parvum-primed mice were reduced by LNMMA treatment (28). This also suggests an interesting correlation between LPS and NO inhibitors as well as the effect of serum on the stimulatory effect of LPS. In another study, the induction of iNOS by LPS was markedly reduced in the absence of fetal calf serum (FCS) (29). A likely explanation for that event is that some serum factor might be required for the induction of iNOS by LPS. A candidate is LPS-binding protein (LBP) which binds to LPS to form an LBP-LPS complex which then binds to the CD14 receptor on the cell membrane (30). The same study reported that induction of NOS by IFN, however, was enhanced in the absence of FCS. This may be related to a possible nonspecific binding of IFN to plasma proteins present in FCS (29). Another study reported that LPS stimulated the induction of NOS in RAW 264.7 macrophages with maximal expression at concentrations of 1-3 $\mu \mathrm{g} / \mathrm{ml}$. This was wholly dependent upon the presence of serum, indicating that serum differentially affected LPS-stimulated NOS induction (31). Such serum factor influence might also represent some of the differences usually seen in the in vitro NO production between murine and human cells, since FCS and human $\mathrm{AB}^{+}$serum, respectively, are used. We are not aware of other reports regarding the possible influence of human $\mathrm{AB}^{+}$serum as seen here for FCS.

We have provided substantial evidence that human cells, when properly stimulated, do produce NO. It should be noted that human macrophages seem to synthesize less NO than those of murine origin, and the biological consequences of this low production are not yet clear. It has been demonstrated that human mononuclear phagocytes can produce iNOS mRNA and protein and, despite this, their ability to generate NO is very low (32). Furthermore, in the present study we show that LPS and antigenic stimulation have different abilities to induce NO production, with results that seem to reduce the effectiveness of LPS as a good stimulus for NO production by human mononuclear cells. In our experiments, LPS did not stimulate any further release of $\mathrm{NO}$ other than that induced by antigenic stimulation.

In addition to iNOS, human monocytes/ macrophages express the cNOS (33). Previous studies have indicated that induction of iNOS expression may be kept suppressed by the endogenous NO level produced by a cNOS enzyme. In cell types possessing both cNOS and iNOS, this may represent a clear paradox (34). In view of these observations, it would be expected that the endogenous NO levels produced by cNOS could suppress iNOS expression in human PBMC and be incriminated for the lower nitrite concentrations released by human cells. In fact, human cells commonly do not exhibit the same capacity for higher NO production as rodent cells and perhaps this phenomenon, in parallel to the differences in LPS-induced iNOS activation in human cells, could explain these distinct results. In conclusion, the effects of NO on the IVG reaction in schistosomiasis are impressive, especially when we begin to realize how NO consis- 
tently mediates the granulomatous response in an irrefutable, although heterogeneous, way.

\section{Acknowledgments}

We wish to thank Shauma Gustavson and Celia A. Almeida for technical assistance.

\section{References}

1. Appleton I, Tomlinson A \& Willoughby DA (1996). Induction of cyclo-oxygenase and nitric oxide synthase in inflammation. Advances in Pharmacology, 35: 27-78.

2. Green SJ \& Nacy CA (1993). Antimicrobial and immunopathologic effects of cytokine-induced nitric oxide synthesis. Current Opinion in Infectious Diseases, 6: 384-396

3. Xie Q-W \& Nathan C (1994). The highoutput nitric oxide pathway: role and regulation. J ournal of Leukocyte Biology, 56: 576-582.

4. Schneemann M, Schoedon G, Hofer S, Blau N, Guerrero L \& Schaffner A (1993). Nitric oxide synthase is not a constituent of the antimicrobial armature of human mononuclear phagocytes. J ournal of Infectious Diseases, 167: 1358-1362.

5. Chen L, Pace J L, Russel SW \& Morrison DC (1996). Altered regulation of inducible nitric oxide synthase expression in macrophages from senescent mice. Infection and Immunity, 64: 4288-4298.

6. MacMicking J, Xie Q-W \& Nathan C (1997). Nitric oxide and macrophage function. Annual Review of Immunology, 15: 323-350.

7. Parra J C, Doughty B, Colley DG \& Gazzinelli G (1992). Human schistosomiasis mansoni: studies on in vitro granuloma modulation. Memórias do Instituto Oswaldo Cruz, 87 (Suppl 5): 79-81.

8. Dougthy BL, Ottesen EA, Nash TE \& Phillips SM (1984). Delayed hypersensitivity granuloma formation around Schistosoma mansoni eggs in vitro. III. Granuloma formation and modulation in human schistosomiasis mansoni. J ournal of Immunology, 133: 993-997.

9. Oliveira DM, Silva-Teixeira DN, Carmo SA \& Goes AM (1998). Role of nitric oxide on human schistosomiasis mansoni: upregulation of in vitro granuloma formation by L-NAME. Nitric Oxide: Biology and Chemistry, 2: 57-65.

10. Katz N, Chaves A \& Pellegrino J (1972). A simple device for quantitative stool thicksmear technique in schistosomiasis mansoni. Revista do Instituto de Medicina Tropical de São Paulo, 14: 397-400.

11. Thorsby G \& Bratlier A (1970). A rapid method for preparation of pure lymphocyte suspensions. In: Terasaki PI (Editor), Histocompatibility Testing. Williams and Wilkins, Baltimore, 665-667.

12. Doughty BL, Goes AM \& Parra J C (1987). Granulomatous hypersensitivity to Schistosoma mansoni egg antigens in human schistosomiasis. I. Granuloma formation and modulation around polyacrylamide antigen-conjugated beads. Memórias do Instituto Oswaldo Cruz, 82: 47-52.

13. Green LC, Wagner DA, Glogowski J , Skipper PL, Wishnok J S \& Tannenbaum SR (1982). Analysis of nitrate, nitrite and $\left[{ }^{15} \mathrm{~N}\right]$ nitrate in biological fluids. Analytical Biochemistry, 126: 131-138.

14. Xiong $\mathrm{H}$, Kawamura $\mathrm{I}$, Nishibori $\mathrm{T} \&$ Mitsuyama M (1996). Suppression of IFN $\gamma$ production from Listeria monocytogenesspecific $T$ cells by endogenously produced nitric oxide. Cellular Immunology, 172: 118-125.

15. Albina JE (1995). On the expression of nitric oxide synthase by human macrophages: why no NO? J ournal of Leukocyte Biology, 58: 643-646.

16. Weinberg J B, Misukonis MA, Shami PJ , Mason SN, Saulus DL, Dittman WA, Wood ER, Smith GK, McDonald B \& Bachus KE (1995). Human mononuclear phagocyte inducible nitric oxide synthase (iNOS): analysis of iNOS mRNA, iNOS protein, biopterin, and nitric oxide production by blood monocytes and peritoneal macrophages. Blood, 86: 1184-1195.

17. Sunderkotter C, Steinbrik K, Goebeler M, Bhaidwaj R \& Sorg C (1994). Macrophages and angiogenesis. Journal of Leukocyte Biology, 55: 410-422.

18. Schmidt HHW \& Walter U (1994). NO at work. Cell, 78: 919-925.

19. Muhl H\& Dinarello CA (1997). Macrophage inflammatory protein- $1 \alpha$ production in lipopolysaccharide-stimulated human adherent blood mononuclear cells is inhibited by the nitric oxide synthase $\mathrm{N}_{\mathrm{G}}$ monomethyl-L-arginine. J ournal of Immunology, 159: 5063-5069.

20. Connor J R, Manning PT, Settle SL, Moore WM, J erome GM, Webber RK, Tjoeng FS \& Currie MG (1995). Suppression of adjuvant-induced arthritis by selective inhibi- tion of inducible nitric oxide synthase. European J ournal of Pharmacology, 273: 1524.

21. Kroncke K-D, Fehsel K \& Kolb-Bachofen V (1995). Inducible nitric oxide synthase and its product nitric oxide, a small molecule with complex biological activities. Biological Chemistry Hoppe-Seyler, 376: 327343.

22. Sriskandan S, Moyes D, Buttery LK, Wilkinson J , Evans TJ , Polak J \& Cohen J (1997). The role of nitric oxide in experimental murine sepsis due to pyrogenic exotoxin A-producing Streptococcus pyogenes. Infection and Immunity, 65: 17671772

23. Messmer UK, Ankarcrona $M$, Nicotera $P$ \& Brune B (1994). P53 expression in nitric oxide-induced apoptosis. FEBS Letters, 355: 23-27.

24. Rees DD (1995). Role of nitric oxide in the vascular dysfunction of septic shock. Biochemical Society Transactions, 23: 10251029.

25. Rees DD, Monkhouse J E \& Moncada S (1996). Inhibitors of NO formation: from organ bath to clinical applications in sepsis. Proceedings of the Scandinavian Physiological Society Meeting, 10-12 May, 1996, Stockholm. Acta Physiologica Scandinavica, 157: 1A-60A.

26. Lorsbach RB, Murphy WJ, Lowenstein CJ , Snyder SH \& Russel SW (1993). Expression of the nitric oxide synthase gene in mouse macrophages activated for tumor cell killing. Molecular basis for the synergy between interferon- $\gamma$ and lipopolysaccharide. J ournal of Biological Chemistry, 268: 1908-1913.

27. Corraliza IM, Soler G, Eichmann K \& Modolell M (1995). Arginase induction by suppressors of nitric oxide synthesis (IL4, IL-10 and PGE2) in murine bone marrow-derived macrophages. Biochemical and Biophysical Research Communications, 206: 667-673.

28. Smith $S R, M a n f r a ~ D$, Davies $L$, Terminelli C, Denhardt G \& Donkin J (1997). Elevated levels of NO in both unchallenged and LPS-challenged $C$. parvum-primed mice are attributable to the activity of a cytokine-inducible isoform of iNOS. J ournal 
of Leukocyte Biology, 61: 24-32.

29. Szabó C, Southan GJ , Wood E, Thiemermann C \& Vane J R (1994). Inhibition by spermine of the induction of nitric oxide synthase in 774.2 macrophages: requirement of a serum factor. British J ournal of Pharmacology, 112: 355-356.

30. Raetz CRH, Ulevitch RJ , Wright SD, Sibley CH, Ding A \& Nathan CF (1991). Gramnegative endotoxin: an extraordinary lipid with profound effects on eukaryotic signal transduction. FASEB J ournal, 5: 26522660.
31. Paul A, Bryant C, Lawson MF, Chilvers ER \& Plevin R (1997). Dissociation of lipopolysaccharide-mediated induction of nitric oxide synthase and inhibition of DNA synthesis in RAW 264.7 macrophages and rat aortic smooth muscle cells. British J oumal of Pharmacology, 120: 1439-1444.

32. Liew FY (1994). Regulation of nitric oxide synthesis in infections and autoimmune diseases. Immunology Letters, 43: 95-98.

33. Reiling $\mathrm{N}$, Ulmer AJ, Duchrow $M$, Ernst M, Flad H \& Hauschildt S (1994). Nitric oxide synthase: mRNA expression of dif- ferent isoforms in human monocytes/ macrophages. European J ournal of Immunology, 24: 1941-1945.

34. Colasanti M, Cavalieri E, Persichini T, Mollace V, Mariotto S, Suzuki H \& Lauro GM (1997). Bacterial lipopolysaccharide plus interferon-gamma elicit a very fast inhibition of a $\mathrm{Ca}^{2+}$-dependent nitric oxide synthase activity in human astrocytoma cells. J oumal of Biological Chemistry, 272: 7582-7585. 